\title{
Prospective study of carmustine wafers in combination with 6-month metronomic temozolomide and radiation therapy in newly diagnosed glioblastoma: preliminary results
}

\author{
Clinical article
}

\author{
Andrea Salmaggi, M.D., Ida Milanesi, M.D., Antonio Silvani, M.D., \\ Paola Gaviani, M.D., Marcello Marchetti, M.D., Laura Fariselli, M.D., \\ Carlo lazzaro Solero, M.D., Carmelo Maccagnano, M.D., Cecilia Casali, M.D., \\ Sara Guzzetti, B.Sc., Bianca Pollo, M.D., Emilio Ciusani, Ph.D., \\ and Francesco DiMeco, M.D.
}

Fondazione IRCCS Istituto Neurologico C. Besta, Milan, Italy

\begin{abstract}
Object. Locoregional chemotherapy with carmustine wafers, positioned at surgery and followed by radiation therapy, has been shown to prolong survival in patients with newly diagnosed glioblastoma, as has concomitant radiochemotherapy with temozolomide. A combination of carmustine wafers with the Stupp treatment regimen has only been investigated in retrospective studies.

Methods. In a single-institution prospective study, the authors assessed 12-month progression-free survival (PFS), toxicity, and overall survival in patients with glioblastoma treated with surgery, carmustine wafers, radiotherapy, and 6-month metronomic temozolomide chemotherapy. Thirty-five patients with de novo glioblastoma, between the ages of 18 and 70 years, and with Karnofsky Performance Scale scores of at least 70, were included in the study. Patients were followed monthly and assessed using MRI every 2 months.

Results. After a median follow-up of 15 months, the median time to tumor progression was 12.5 months and median survival was 17.8 months. Due to toxicity (mostly hematological), 7 patients had to prematurely stop temozolomide treatment. Twenty-two patients developed Grade $3 \mathrm{CD}^{+}$lymphocytopenia. Three patients developed oral-esophageal candidiasis, 2 developed pneumonia, and 1 developed a dorsolumbar zoster. Early intracranial hypertension was observed in 1 patient, and 1 was treated empirically for suspected brain abscess. One patient died of Legionella pneumonia soon after repeat surgery.

Conclusions. Overall, this treatment schedule produced promising results in terms of PFS without a marked increase in toxicities as compared with the Stupp regimen. However, the gain in median survival using this schedule was less clear. Only prospective comparative trials will determine whether these preliminary results will translate into a long-term survival advantage with an acceptable toxicity profile.
\end{abstract}

(http://thejns.org/doi/abs/10.3171/2012.12.JNS111893)

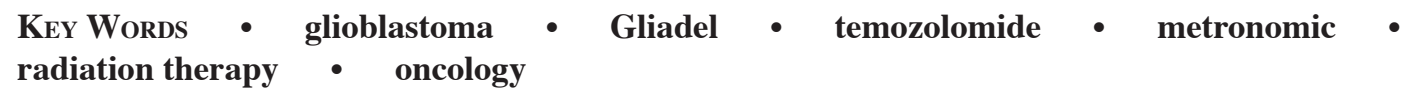

$\mathrm{T}$ HE median survival of patients with newly diagnosed glioblastoma treated according to the Stupp treatment protocol is 14.6 months..$^{25}$ This approach has been supported by the results of a large Phase III trial, thus becoming the standard of therapy in newly diagnosed glioblastoma ${ }^{24}$ despite a long-standing practice of therapy with a number of chemotherapeutic agents and with

Abbreviations used in this paper: CTCAE = Common Terminology Criteria for Adverse Events; KPS = Karnofsky Performance Scale; MGMT = O-6-methylguanine-DNA methyltransferase; PCR = polymerase chain reaction; $\mathrm{PFS}=$ progression-free survival; RPA $=$ recursive partitioning analysis. evidence of efficacy suggested only by meta-analysis. ${ }^{12}$ However, glioblastoma invariably recurs, most frequently in the proximity of the original tumor site, and more rarely at a distance or as a multifocal or diffuse tumor. ${ }^{5}$ After recurrence, median survival time does not exceed 9-10 months, even in the setting of Phase II trials with novel agents such as bevacizumab. ${ }^{8,17}$

Locoregional chemotherapy has been assessed with the use of carmustine wafers (Gliadel, manufactured by Eisai) positioned by the neurosurgeon in the surgical cavity. After evidence of increased survival after recurrence following repeat surgery and carmustine wafers, as compared with repeat surgery alone, ${ }^{4}$ a trial was performed in 
$2003 .^{30}$ The results of this trial suggested some survival advantage of using carmustine wafers combined with radiotherapy in newly diagnosed glioblastoma, but without the addition of temozolomide. ${ }^{30}$

To increase antitumoral activity in the treatment protocols, several studies have addressed the feasibility of combining carmustine wafers with the Stupp treatment protocol, with results showing a variable profile of tolerability and toxicity and possibly interesting effects on PFS and median survival time., $3,11,14,17,18,21$ Multimodal approaches are increasingly being advocated in the treatment of aggressive tumors, including glioblastoma. The reasons for this advocacy include the presence within the tumor of heterogeneous genetic abnormalities ${ }^{27}$ as well as the inability of the presently adopted treatment schedules to effectively prolong PFS and overall survival in a clinically meaningful way.

In the present study we investigated the tolerability and possible efficacy of local carmustine wafers in combination with radiotherapy and prolonged metronomic temozolomide chemotherapy in patients with newly diagnosed glioblastoma who had undergone resection. The rationale for choosing a metronomic schedule of temozolomide is a possible effect on MGMT activity even in patients with a nonmethylated MGMT promoter, ${ }^{27,28}$ as well as a possible antiangiogenic effect in the context of minimal residual disease after surgery. ${ }^{10}$ Moreover, beginning chemotherapy 2 weeks after surgery may result in increased killing of residual tumor cells before the beginning of radiation therapy, adding systemic chemotherapy to the effects of carmustine released locally by surgically placed implants. We report the preliminary data of a prospective single-institution Phase II study involving carmustine wafers at surgery combined with radiation therapy and protracted metronomic temozolomide in patients with newly diagnosed glioblastoma.

\section{Methods}

\section{Study Population}

The study protocol was approved by the Ethical Committee of Fondazione IRCCS Istituto Neurologico C. Besta. The design of the study was a 2-phase investigation according to the recommendations of Simon; ${ }^{23}$ we assumed a clinically relevant result to be an increase of $15 \%$ in the proportion of patients free from disease progression or recurrence at 12 months as compared with the results of Stupp et al..$^{25}(27 \%)$, with a 5\% probability of a Type I error and a 10\% probability of a Type II error.

Thirty-five patients were included in the first part of the study. Inclusion criteria were as follows: supratentorial, newly diagnosed glioblastoma; gross-total or subtotal resection (degree of resection was defined as subtotal if residual nodular enhancement was noted on postoperative MRI performed within 48 hours after surgery); age range 18-70 years; KPS score of 70 or higher; normal liver, kidney, and bone marrow functions; and no concomitant infections. Life expectancy had to be longer than 3 months. Exclusion criteria were as follows: no histological analysis available; previous biopsy procedure performed; multifocal or infratentorial glioblastoma; age younger than 18 years and older than 70 years; KPS score less than 70; concomitant infections; and altered kidney, liver, or bone marrow functions.

\section{Treatment Protocol}

The proportion of patients free from recurrence at 12 months was the primary endpoint of the study. Secondary endpoints included toxicity and median survival time. After obtaining informed consent, the treatment protocol included surgery with carmustine wafer positioning; after 7-15 days from surgery, chemotherapy was started with temozolomide at a dose of $75 \mathrm{mg} / \mathrm{m}^{2}$ daily, for up to 6 months or until development of toxicity or confirmed disease progression.

Standard external beam radiation therapy was delivered in 30 sessions, starting within 30 days from surgery for a total dose of $60 \mathrm{~Gy}$. Prophylaxis with trimethoprim and sulfamethoxazole was delivered to all patients for the duration of the chemotherapy treatment. Patients were assessed clinically every month and underwent contrastenhanced brain MRI every 2 months.

Disease progression was evaluated according to the criteria of McDonald et al. ${ }^{12}$ In cases of suspected early progression (within 3 months from the end of radiation therapy), repeat MRI was performed at 45 days to rule out pseudoprogression. Complete blood cell count was assessed weekly, liver function every 2 weeks, and lymphocyte subsets (CD4 and CD8) and creatinine levels every 3 weeks. Toxicities were defined according to CTCAE version 3.0. In cases of protracted Grade I toxicity, the temozolomide dose was reduced by $25 \%$.

\section{MGMT Promoter Methylation Status}

Tumor genomic DNA was extracted by tissue using the PureLink Genomic DNA Mini Kit (Invitrogen) according to the manufacturer's instructions for formalin-fixed, paraffin-embedded tissue lysate and stored at $4^{\circ} \mathrm{C}$. The methylation status was assessed by the MethylEasy DNA Bisulphite Modification Kit (Diagenode). Briefly, $2.2 \mu \mathrm{l}$ of $3 \mathrm{M} \mathrm{NaOH}$ was added to $4 \mu \mathrm{g}$ DNA in $20 \mu \mathrm{l}$ solution, mixed gently, and incubated for 15 minutes at $37^{\circ} \mathrm{C}$. We then added $220 \mu \mathrm{l}$ of modification reagent and overlayed the solution with $200 \mu \mathrm{l}$ of mineral oil. The DNA was than incubated at $55^{\circ} \mathrm{C}$ for 16 hours. Four microliters of the converted DNA sample was used in each PCR reaction. Methylated-specific PCR was performed using primers designed for unmethylated or methylated DNA. ${ }^{26}$ Two independent PCR reactions were obtained to confirm results.

Methylated-specific PCR products were analyzed on an ABI Prism 3130 (Applied Biosystems). Two microliters of methylated- or unmethylated-specific DNA fragment was added to a mix containing $10 \mu \mathrm{l}$ of formamide and $0.3 \mu \mathrm{l}$ of Gene Scan-500 LIZ Size Standard Marker (Applied Biosystems). Peaks of 81 base pairs (Peak 1) and 96 base pairs (Peak 2), corresponding to methylated and unmethylated DNA, respectively, were detected using a separation matrix for the 3130 genetic analyzer (3130 POP-7, Applied Biosystems). Data were analyzed with the Gene Scan program for fragment analysis (ABI Prism 
3130, Applied Biosystems). The ratio between height peaks (Peak 1/Peak 2) provides an estimation of allelic ratio. Values greater than or equal to 0.1 indicate MGMT promoter methylated status, while lower values indicate unmethylated status of the promoter.

\section{Statistical Analysis}

The analysis of PFS and median survival was performed using Kaplan-Meier survival curves. Descriptive statistics were used for evaluation of toxicities. Level of significance was set at $\mathrm{p}<0.05$.

\section{Results}

\section{Clinical Data}

Thirty-five patients have been recruited into the study to date. The clinical features of these patients are detailed in Table 1. Overall, there were 25 males and 10 females, with ages ranging from 27 to 70 (median 58). The RPA class was IV in 26 cases and III in 9 cases. Median KPS score was 80 . The median follow-up was 15 months (range 8-30 months). One patient was lost to follow-up 1 month after surgery for withdrawal of consent. Of the 32 patients with a follow-up longer than or equal to 12 months (including patients who died within 12 months), 21 experienced tumor progression, with $50 \%$ PFS at 12 months (Fig. 1). The median time to tumor progression in the entire cohort of patients was 12.5 months (Fig. 1).

Overall, 21 patients have shown disease progression, and 18 (12 males and 6 females) have died (at 8, 8, 10, $10,13,13,13,14,15,15,16,16,18,18,19,21,24$, and 28 months). Sixteen of these 18 patients died due to tumor progression, while 1 male patient died suddenly at 6 months after tumor progression (necropsy was not performed), and 1 patient died at 13 months after repeat surgery for Legionella pneumonia. Disease progression took place in proximity of the original surgery in 19 cases, at a distant supratentorial site in 1 case, and in the form of diffuse leptomeningeal dissemination in 1 case.

Treatment at progression included repeat surgery and second-line chemotherapy in 5 cases, whereas secondline chemotherapy was delivered in 8 cases (in 2 cases with bevacizumab and in 6 with fotemustine). Repeat surgery was performed in 3 patients, who did not continue other chemotherapy due to poor clinical conditions. Four patients were in poor general condition and were followed with supportive therapy only. One patient was treated by CyberKnife radiosurgery followed by second-line chemotherapy with fotemustine.

Median survival was 17.8 months (Fig. 2), and was not significantly different if calculated in the patients with follow-up equal to or longer than 12 months (data not shown). Significant differences were noted in time to tumor progression at univariate analysis according to RPA class ( $\mathrm{p}$ $=0.03, \log$ rank test), while no significant differences were observed in total versus subtotal resection, KPS score of 70 versus a higher score, and MGMT promoter methylation status (8 methylated vs 22 nonmethylated). At univariate analysis, survival time in patients was significantly longer, at a statistically borderline level, only in patients with a methylated MGMT promoter $(\mathrm{p}=0.049)$, whereas it was not influenced by KPS score, RPA class, or $\mathrm{CD}^{+}{ }^{+}$lymphocytopenia development (data not shown).

\section{Toxicity Data}

The toxicity profile of the treatment schedule is reported in Table 2. Overall, 7 patients had to prematurely stop temozolomide therapy due to toxicity; 1 patient developed Grade 4 thrombocytopenia during radiotherapy, and 1 developed nephrotoxicity (CTCAE Grade 3). Two other patients developed CTCAE Grade 4 thrombocytopenia after 3 months of therapy, and 1 developed CTCAE Grade 4 leukopenia and thrombocytopenia at 2 months of therapy. One patient developed Grade 2 liver toxicity, with alteration of laboratory tests Grade 4 for bilirubin and Grade 3 for alanine aminotransferase and aspartate aminotransferase, after 45 days of chemotherapy. Finally, 1 patient developed Grade 4 thrombocytopenia after 5 months of chemotherapy.

Overall, 5 patients had to reduce their temozolomide dose by $25 \%$, due to liver toxicity in 1 patient, leukopenia in 3 patients, and thrombocytopenia in 1 patient. A dose reduction by $25 \%$ was planned in cases of persistent Grade 1 toxicity (for platelets) and Grade 2 toxicity (for neutrophils) after obtaining 2 blood samples 1 week apart. One female patient developed a late Grade 4 leukopenia just after the end of the 6-month temozolomide therapy, which promptly receded with growth factor administration. Three patients did not complete the planned treatment with temozolomide due to early tumor progression.

$\mathrm{CD}^{+}$lymphocytopenia occurred in 22 of 34 patients. In all cases, this was Grade 3 lymphocytopenia and developed after at least 2 months of chemotherapy, persisting at follow-up. As noted in Table 2, 3 patients developed oral-esophageal candidiasis, which was managed with oral antimycotic therapy, and 1 patient developed pneumonia that receded with antibiotic therapy. One patient developed a dorsolumbar herpes zoster infection after 6 months of chemotherapy; she was treated with antiviral therapy and had moderate residual postherpetic pain. One patient developed pneumonia soon after the first fotemustine cycle following repeat surgery. This infection was successfully managed with antibiotics but required hospitalization. One patient died of Legionella pneumonia after repeat surgery. One patient was treated empirically for suspected brain abscess beginning 10 days after surgery, with a delay in radiation therapy and chemotherapy of 14 days and no subsequent complications.

Deep venous thrombosis $(n=4)$ and/or pulmonary embolism ( $n=1)$ occurred in 4 cases, always during metronomic chemotherapy administration. Other complications included multiple vertebral collapses likely due to steroid treatment in 1 male patient and development of diabetes in 5 cases ( 3 of whom were insulin dependent). Seizures were present at diagnosis or occurred during follow-up in 21 of 33 patients, and were well controlled by medical treatment. Only 1 patient developed postoperative intracranial hypertension, which was managed by dexamethasone and mannitol infusion. A review of the pattern of toxicities reported in other studies combining locoregional carmustine with the Stupp protocol is shown in Table 3. 
TABLE 1: Clinical features of patients included in the study

\begin{tabular}{|c|c|c|c|c|c|}
\hline Case No. & Age (yrs), Sex & Tumor Site & KPS Score & Resection & RPA Class \\
\hline 1 & $64, \mathrm{~F}$ & rt temporal & 70 & total & IV \\
\hline 2 & $45, \mathrm{~F}$ & It frontal & 90 & total & III \\
\hline 3 & $55, \mathrm{M}$ & It temporal & 70 & subtotal & IV \\
\hline 4 & $47, \mathrm{M}$ & It parietal & 80 & subtotal & IV \\
\hline 5 & $52, \mathrm{~F}$ & rt temporooccipital & 90 & total & IV \\
\hline 6 & $40, M$ & rt frontal & 90 & total & III \\
\hline 7 & $61, \mathrm{~F}$ & rt temporal & 80 & total & IV \\
\hline 8 & $61, \mathrm{M}$ & rt parietal & 70 & subtotal & IV \\
\hline 9 & $54, \mathrm{~F}$ & It frontoparietal & 70 & subtotal & IV \\
\hline 10 & $50, \mathrm{M}$ & rt frontal & 80 & total & III \\
\hline 11 & $65, \mathrm{M}$ & It frontal & 70 & total & IV \\
\hline 12 & $65, \mathrm{~F}$ & rt frontal & 70 & total & IV \\
\hline 13 & $68, M$ & rt frontal & 80 & subtotal & IV \\
\hline 14 & $52, \mathrm{~F}$ & It temporal & 90 & total & IV \\
\hline 15 & $27, \mathrm{M}$ & It frontal & 90 & total & III \\
\hline 16 & $60, M$ & It temporal & 70 & total & IV \\
\hline 17 & $45, M$ & rt frontal & 80 & total & III \\
\hline 18 & $58, \mathrm{M}$ & rt frontal & 80 & total & IV \\
\hline 19 & $57, \mathrm{M}$ & It frontoparietal & 80 & total & III \\
\hline 20 & $49, \mathrm{M}$ & rt tempoparietal & 70 & total & IV \\
\hline 21 & $67, \mathrm{M}$ & rt tempoparietal & 70 & subtotal & IV \\
\hline 22 & $63, M$ & It temporal & 80 & subtotal & III \\
\hline 23 & $48, \mathrm{~F}$ & It parietal & 70 & subtotal & IV \\
\hline 24 & $67, \mathrm{~F}$ & rt frontotemporal & 90 & total & III \\
\hline 25 & $50, \mathrm{M}$ & It frontal & 00 & total & IV \\
\hline 26 & $63, \mathrm{M}$ & rt temporal & 00 & total & III \\
\hline 27 & $49, \mathrm{M}$ & rt frontal & 80 & total & IV \\
\hline 28 & $58, \mathrm{M}$ & rt parietal & 90 & total & IV \\
\hline 29 & $62, \mathrm{M}$ & rt temporal & 70 & total & IV \\
\hline 30 & $70, M$ & rt temporal & 90 & total & IV \\
\hline 31 & $57, \mathrm{M}$ & It temporal & 90 & subtotal & IV \\
\hline 32 & $66, M$ & It parietal & 90 & total & IV \\
\hline 33 & $61, \mathrm{M}$ & rt frontotemporal & 60 & total & IV \\
\hline 34 & $70, \mathrm{M}$ & It frontal & 70 & subtotal & IV \\
\hline 35 & $53, F$ & It temporal & 80 & total & IV \\
\hline
\end{tabular}

\section{Discussion}

This study shows that a high proportion of patients with newly diagnosed glioblastoma were free from disease recurrence at 12 months. A number of mostly retrospective studies have investigated the tolerability and efficacy of the combination of the Stupp protocol with carmustine wafers locoregionally. In these studies, tolerability data are good and some effectiveness of the treatment appears to be likely. $3,12,15,17,18,21$

As expected in Phase II studies, the rates of PFS at 12 months vary significantly among studies due to a number of factors. Among these factors, differences in patient populations are the most relevant, together with the variable type of tumor resection. Overall, the percentage of patients free from disease recurrence at 12 months varies from $29 \%{ }^{10}$ to $35 \%{ }^{11}$ to $40 \% .^{19}$

The $50 \%$ of patients free from disease progression at 12 months - in the only prospective study reported so far to our knowledge-exceeds the best results obtained in retrospective studies by combining carmustine wafers and the Stupp protocol, except for the data reported by Noël et al. ${ }^{17}$ These encouraging results were obtained with a treatment schedule inducing marked lymphocytopenia; of note, Sampson and colleagues ${ }^{22}$ recently reported that such schedules may paradoxically enhance tumor-specific immune responses in patients with glioblastoma.

Despite this improvement, however, patients continue to progress and die of their disease, and median survival in recurrent glioblastoma does not exceed 10 months, 


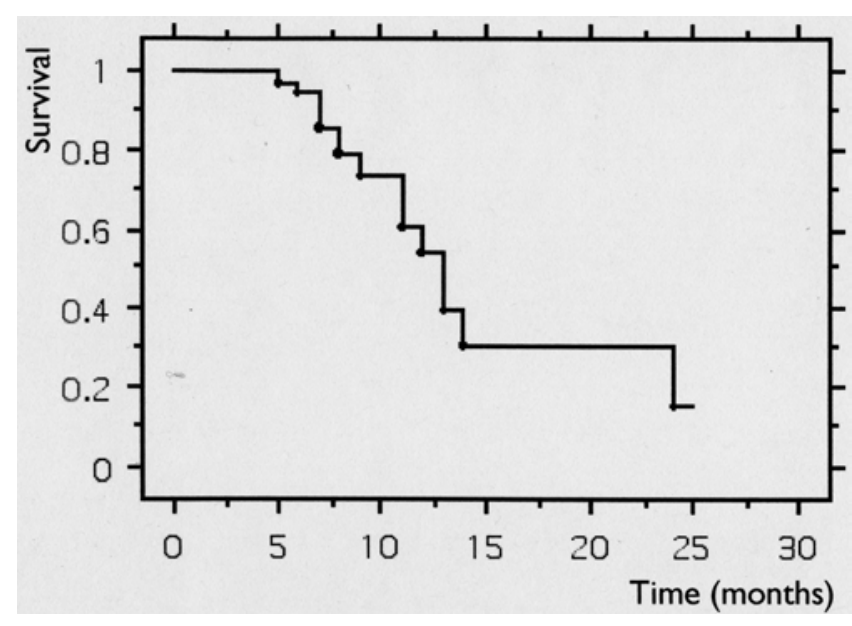

Fig. 1. Graph of the time to tumor progression in the entire cohort of patients.

even in bevacizumab-treated patients. ${ }^{29}$ Because the gain in survival is more debatable, as compared with the trial of Stupp et al., it is extremely important to address toxicity issues. From this point of view, the present multimodal schedule of treatment did not appear to show a meaningfully higher burden of side effects as compared with a combination of carmustine wafers with the Stupp protocol. Notably, the proportion of patients who had to interrupt treatment because of toxicities (7 of 35, 20\%) was not higher than that reported in the trial of Stupp et al., ${ }^{25}$ in which $22 \%$ of patients could not start the adjuvant phase of temozolomide chemotherapy (only 4\% for early progression). The percentage of patients suffering from deep venous thrombosis/pulmonary embolism was also similar to that reported in previous works. ${ }^{13}$

Concerning infections, 1 case of herpes zoster, 2 cases of pneumonia of undetermined origin, and 3 cases of nondisseminated candidiasis were detected, and all were responsive to standard treatment. One patient died due to Legionella pneumonia 1 week after repeat surgery; his total lymphocyte count was normal. Protracted prophylaxis with trimethoprim and sulfamethoxazole may have prevented the occurrence of Pneumocystis infections

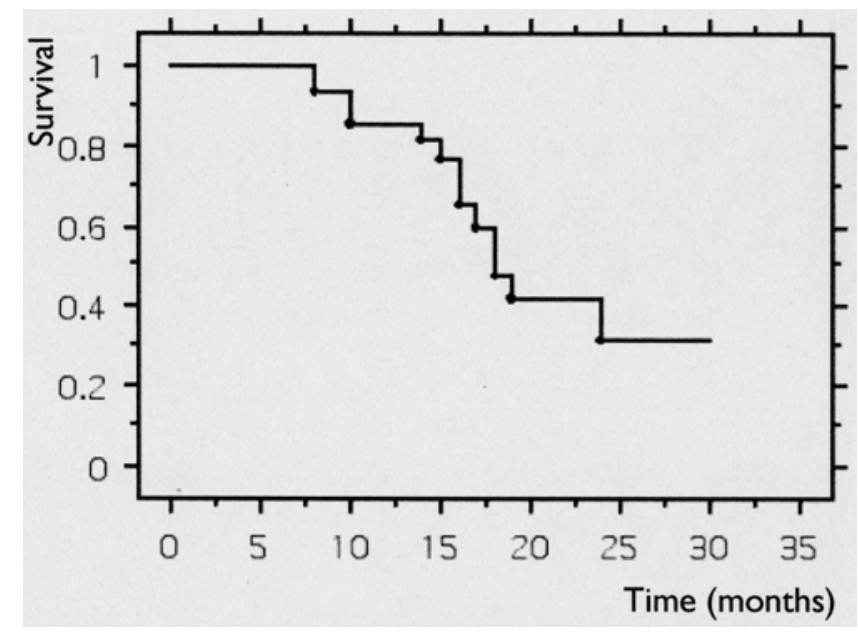

FIG. 2. Graph of survival time in the entire cohort of patients. (which did not take place in this cohort), even if we cannot exclude that it may partly be responsible for the observed cases of liver and kidney toxicities.

Although the relatively low frequency of severe infections in patients displaying marked lymphocytopenia may be surprising at first glance, previously reported data have demonstrated that in long-term survivors of childhood malignancies treated using chemotherapy, profound $\mathrm{CD}^{+}{ }^{+}$reduction was detected in nearly $10 \%$, and that in those patients no increase in severe infections took place. ${ }^{2}$ In fact, some evidence exists showing that after intensive chemotherapy delivered for leukemia, residual $\mathrm{T}$ cells may show hyperactivity with a broad cytokine release, thus partly compensating for the profound numeric loss in counteracting possible infections. ${ }^{7}$ The infections developing in our patients were not limited solely to those patients with $\mathrm{CD}^{+}$depletion. We did not observe intracranial infections, except for 1 case of a suspected brain abscess that was managed conservatively by antibiotic treatment due to empirical evidence.

Diabetes remained a clinical problem in our patients, possibly due to a slow tapering of steroid dose after surgery to avoid intracranial hypertension (which actually took place in only 1 case). Seizures did not represent a clinical problem, with a frequency similar to that reported in the literature and no problems in their management. One patient died suddenly, a few weeks after radiological evidence of tumor progression, in the context of stable neurological conditions; a necropsy was not performed and the clinically most likely causes include cardiac disease with rhythm alteration or pulmonary embolism.

A number of mostly retrospective studies have been published on the combination of locoregional carmustine and postoperative radiotherapy with concomitant temozolomide followed by adjuvant temozolomide. These data, including toxicity analysis, have been recently reported in a review ${ }^{6}$ and are summarized in Table 3. Overall, the frequency and severity of side effects are highly variable, as expected considering the heterogeneity of the patient populations. However, toxicities not related to local complications (such as brain abscess, intracranial hypertension, and seizures) do not appear to be substantially increased as compared with those encountered in patients included in the trial of Stupp et al., ${ }^{25}$ while side effects were not described in detail in the trial of Westphal et al. ${ }^{30}$

Although putative additive toxicities may be overestimated due to the prospective nature of our study, we observed an increase in non-CNS infections in our patient cohort. The lack of statistically significant differences in the proportion of progression-free patients and in survival time according to age, KPS score, and extent of resection is likely due to either selection criteria or to the low number of patients. Only borderline statistical significance was achieved for survival time considering the methylation status of the MGMT promoter.

In our cohort of patients, the adopted schedule of treatment did not appear to modify the pattern of recurrence. In fact, recurrence occurred in the great majority of cases close to the original tumor site, and only in 2 cases did it occur at a distance. Thus, metronomic chemotherapy with temozolomide, which supposedly may have 
TABLE 2: Side effects*

\begin{tabular}{|c|c|c|c|c|c|c|}
\hline $\begin{array}{l}\text { Case } \\
\text { No. }\end{array}$ & $\begin{array}{l}\text { Early Intracranial } \\
\text { Hypertension }\end{array}$ & $\begin{array}{c}\text { Brain } \\
\text { Abscess }\end{array}$ & Other Infections & DVT/TE & $\begin{array}{l}\text { Other } \\
\text { Toxicities }\end{array}$ & Chemotherapy Stopped \\
\hline 1 & no & no & no & & & yes (progression) \\
\hline 2 & no & no & no & & & \\
\hline 3 & yes & no & no & & & yes (kidney) \\
\hline 4 & no & no & no & & & yes (decrease in platelets) \\
\hline 5 & no & no & dorsolumbar herpes zoster & DVT (Day 90) & & \\
\hline 6 & no & no & no & & kidney & $\begin{array}{l}\text { yes (decrease in white } \\
\text { blood cells) }\end{array}$ \\
\hline 7 & no & no & no & & & \\
\hline 8 & no & no & no & & & \\
\hline 9 & no & yes & no & & & yes (decrease in platelets) \\
\hline 10 & no & no & oral candidiasis & & & \\
\hline 11 & no & no & no & & & \\
\hline 12 & no & no & oral candidiasis & VTE (Day 60) & & yes (progression) \\
\hline 13 & no & no & no & & & \\
\hline 14 & no & no & no & & & \\
\hline 15 & no & no & no & & & withdrew consent \\
\hline 16 & no & no & no & & & \\
\hline 17 & no & no & no & & & \\
\hline 18 & no & no & no & & & \\
\hline 19 & no & no & pneumonitis & & & \\
\hline 20 & no & no & no & & & \\
\hline 21 & no & no & no & DVT & & \\
\hline 22 & no & no & no & & & \\
\hline 23 & no & no & oral candidiasis & & & yes (progression) \\
\hline 24 & no & no & no & & & \\
\hline 25 & no & no & no & & & \\
\hline 26 & no & no & no & & & yes (decrease in platelets) \\
\hline 27 & no & no & no & & & \\
\hline 28 & no & no & no & & & \\
\hline 29 & no & no & no & & & yes (liver) \\
\hline 30 & no & no & no & DVT & & \\
\hline 31 & no & no & no & & & yes (decrease in platelets) \\
\hline 32 & no & no & no & & liver & yes (progression) \\
\hline 33 & no & no & legionellosis & & & \\
\hline 34 & no & no & no & & & yes (kidney) \\
\hline 35 & no & no & no & & & yes (decrease in platelets) \\
\hline
\end{tabular}

* DVT = deep venous thrombosis; TE = thromboembolism; VTE = venous TE.

an antiangiogenic effect, did not lead to an increase in tumor infiltration or dissemination as compared with the general pattern in glioblastoma. ${ }^{5,18}$ It can be speculated that the remarkable efficacy of the schedule in prolonging PFS may be due to an increased inhibiting activity of MGMT by daily temozolomide, even if further work is needed to verify this hypothesis.

Despite very promising effects on PFS, median survival time was less significantly improved in our study; this is partly in agreement with the recently published results of the Radiation Therapy Oncology Group 0525 trial, which has shown a lack of effect of dose-intensified temozolomide on survival time, despite a slight improvement in PFS. ${ }^{9}$ In this study, the median survival time was longer if compared with data from the trial of Stupp et al. ${ }^{25}$ This may partly be due to inclusion of selected patients in our study (that is, those with total or subtotal resection); if we compare our median survival time (17.8 months), with the survival time of patients in the Stupp et al. trial (15.8 months) excluding those undergoing biopsy (126 with partial resection and 113 with total resection), the difference is approximately 9 weeks.

It remains to be definitively assessed whether the increase in PFS after the first diagnosis and the overall 
Carmustine, temozolomide, and radiation therapy in glioblastoma

TABLE 3: Profile of toxicity in patients with malignant glioma treated using carmustine implants followed by concomitant radiation therapy and chemotherapy*

\begin{tabular}{|c|c|c|c|c|c|}
\hline Authors \& Year & Study Type & Years of Study & $\begin{array}{c}\text { No. of } \\
\text { Patients }\end{array}$ & Adverse Events & $\begin{array}{l}\text { Toxicity } \\
\text { Grade }\end{array}$ \\
\hline \multirow[t]{5}{*}{ Ryken et al., 2008} & \multirow[t]{5}{*}{ prospective Phase II study } & \multirow[t]{5}{*}{2007} & \multirow[t]{5}{*}{21} & death for multiorgan failure (4.8\%) & 5 \\
\hline & & & & pulmonary embolism (5\%) & 4 \\
\hline & & & & nausea $(5 \%)$ & 4 \\
\hline & & & & liver toxicity $(10 \%)$ & 3 \\
\hline & & & & thrombocytopenia (5\%) & 3 \\
\hline \multirow[t]{8}{*}{ McGirt et al., 2009} & \multirow[t]{8}{*}{ retrospective } & \multirow[t]{8}{*}{ 2004-2006 } & \multirow[t]{8}{*}{33} & myelosuppression $(21 \%)$ & \\
\hline & & & & platelets $(15 \%)$ & $4(12 \%)$ \\
\hline & & & & neutrophils $(6 \%)$ & 2 \\
\hline & & & & seizures $(6 \%)$ & NR \\
\hline & & & & brain edema $(3 \%)$ & NR \\
\hline & & & & DVT (3\%) & NR \\
\hline & & & & pulmonary embolism (10\%) & NR \\
\hline & & & & infection $(3 \%)$ & NR \\
\hline \multirow[t]{2}{*}{ Pan et al., 2008} & \multirow[t]{2}{*}{ retrospective } & \multirow[t]{2}{*}{$2003-2005$} & \multirow[t]{2}{*}{21} & cerebritis $(10 \%)$ & 3 \\
\hline & & & & steroid psychosis $(5 \%)$ & 3 \\
\hline \multirow[t]{4}{*}{ Menei at al., 2010} & \multirow[t]{4}{*}{ retrospective } & \multirow[t]{4}{*}{$2005-2006$} & \multirow[t]{4}{*}{83} & seizure $(4.8 \%)$ & NR \\
\hline & & & & intracranial hypertension $(9.6 \%)$ & 4 \\
\hline & & & & cyst of aseptic abscess (2.4\%) & NR \\
\hline & & & & septic abscess (6\%) & NR \\
\hline Noël et al., 2012 & retrospective & $2007-2008$ & 28 & myelosuppression (14\%) & $3-4$ \\
\hline \multirow[t]{6}{*}{ Bock et al., 2010} & \multirow[t]{6}{*}{ retrospective } & \multirow[t]{6}{*}{$2005-2008$} & \multirow[t]{6}{*}{44} & seizures $(16 \%)$ & NR \\
\hline & & & & intracranial abscess ( $5 \%)$ & NR \\
\hline & & & & brain edema $(25 \%)$ & NR \\
\hline & & & & myelosuppression (7\%) & $3-4$ \\
\hline & & & & DVT $(9 \%)$ & NR \\
\hline & & & & 1 patient died of pulmonary embolism & \\
\hline \multirow[t]{4}{*}{ La Rocca \& Mehdorn, 2009} & \multirow[t]{4}{*}{ Phase II study of safety \& efficacy } & \multirow[t]{4}{*}{$2003-2005$} & \multirow[t]{4}{*}{41} & sterile brain abscess (1) & $1-4$ \\
\hline & & & & hematological (14\%) & $1-4$ \\
\hline & & & & aplastic anemia $(2 \%)$ & NR \\
\hline & & & & pneumonia (4\%) & $1-4$ \\
\hline \multirow[t]{7}{*}{ Affronti et al., 2009} & retrospective RPA & $2000-2005$ & 36 & seizure $(11 \%)$ & $1-4$ \\
\hline & & & & infection (11\%) & $1-4$ \\
\hline & & & & pseudomeningocele & NR \\
\hline & & & & headache $(5 \%)$ & 1 \\
\hline & & & & hematological (30\%) & $1-4$ \\
\hline & & & & gastrointestinal (22\%) & $2-4$ \\
\hline & & & & venous thromboembolism (11\%) & NR \\
\hline Salvati et al., 2011 & retrospective & $2006-2008$ & 32 & no Grade 3-4 hematological toxicities & \\
\hline Stupp et al., 2005 & randomized Phase III study & $2000-2002$ & 284 & leukopenia (7\%) & $3-4$ \\
\hline & & & & thrombocytopenia (12\%) & $3-4$ \\
\hline Westphal et al., 2003 & prospective randomized double blind & 1997-1999 & 120 & intracranial hypertension $(9.2 \%)$ & NR \\
\hline
\end{tabular}

* Data reported as control in patients treated with radiotherapy and temozolomide (Stupp et al.) or with radiotherapy and locoregional carmustine (Westphal et al.). Abbreviation: NR = not reported.

$\dagger$ According to the CTCAE criteria. 
acceptable toxicity profile will translate into a definite increase in median survival time, in the absence of longterm side effects ${ }^{16}$ However, the preliminary data from this study are encouraging. For this reason further studies are warranted.

\section{Conclusions}

Level A evidence, derived from randomized controlled Phase III trials, is present for locoregional chemotherapy with carmustine wafers, positioned at surgery and followed by radiotherapy, as well as for concomitant radiochemotherapy with temozolomide. However, the combination of carmustine wafers with the Stupp regimen has only been investigated in retrospective studies, and it is doubtful whether this association leads to an increase in survival without relevant side effects. This prospective study has investigated efficacy (with a primary end point of PFS at 12 months), toxicity, and overall survival in 35 patients with newly diagnosed glioblastoma treated by surgery, carmustine wafers, radiotherapy, and 6-month metronomic temozolomide chemotherapy, with monthly clinical evaluations and MRI every 2 months.

Among inclusion criteria, a KPS score of 70 or higher and an age between 18 and 70 years were requested. Median time to tumor progression was 12.5 months, and median survival was 17.8 months after a median follow-up of 15 months. Early temozolomide discontinuation took place in 7 patients, and there were Grade 3 infections in 4 patients. One patient died from Legionella pneumonia after repeat surgery. The PFS at 12 months in this study compares favorably with data present in the literature concerning single-treatment modalities, although the results for survival are less convincing. There is a need for prospective comparative trials to define more accurately the risk:benefit ratio of these 2 treatment schedules.

\section{Disclosure}

Dr. DiMeco has received support from Eisai for non-studyrelated clinical or research efforts.

Author contributions to the study and manuscript preparation include the following. Conception and design: Salmaggi, Milanesi, Silvani, Gaviani, Marchetti, Fariselli, Casali, Pollo, DiMeco. Acquisition of data: Salmaggi, Milanesi, Silvani, Gaviani, Marchetti, Maccagnano, Casali, Guzzetti, Ciusani, DiMeco. Analysis and interpretation of data: Salmaggi, Silvani, Gaviani, Marchetti, Fariselli, Maccagnano, Casali, Pollo, Ciusani, Dimeco. Drafting the article: Salmaggi, Pollo. Critically revising the article: all authors. Reviewed submitted version of manuscript: all authors. Approved the final version of the manuscript on behalf of all authors: Salmaggi. Statistical analysis: Ciusani. Study supervision: Solero, DiMeco.

\section{References}

1. Affronti ML, Heery CR, Herndon JE II, Rich JN, Reardon DA, Desjardins A, et al: Overall survival of newly diagnosed glioblastoma patients receiving carmustine wafers followed by radiation and concurrent temozolomide plus rotational multiagent chemotherapy. Cancer 115:3501-3511, 2009

2. Azuma E, Nagai M, Qi J, Umemoto M, Hirayama M, Kumamoto $\mathrm{T}$, et al: $\mathrm{CD}^{+} \mathrm{T}$-lymphocytopenia in long-term survivors following intensive chemotherapy in childhood cancers. Med Pediatr Oncol 30:40-45, 1998
3. Bock HC, Puchner MJ, Lohmann F, Schütze M, Koll S, Ketter $\mathrm{R}$, et al: First-line treatment of malignant glioma with carmustine implants followed by concomitant radiochemotherapy: a multicenter experience. Neurosurg Rev 33:441-449, 2010

4. Brem H, Piantadosi S, Burger PC, Walker M, Selker R, Vick NA, et al: Placebo-controlled trial of safety and efficacy of intraoperative controlled delivery by biodegradable polymers of chemotherapy for recurrent gliomas. Lancet 345:1008-1012, 1995

5. Chamberlain MC: Radiographic patterns of relapse in glioblastoma. J Neurooncol 101:319-323, 2011

6. Dixit S, Hingorani M, Achawal S, Scott I: The sequential use of carmustine wafers (Gliadel®) and post-operative radiotherapy with concomitant temozolomide followed by adjuvant temozolomide: a clinical review. Br J Neurosurg 25:459-469, 2011

7. Ersvaer E, Hampson P, Hatfield K, Ulvestad E, Wendelbo Ø, Lord JM, et al: T cells remaining after intensive chemotherapy for acute myelogenous leukemia show a broad cytokine release profile including high levels of interferon-gamma that can be further increased by a novel protein kinase $\mathrm{C}$ agonist PEP005. Cancer Immunol Immunother 56:913-925, 2007

8. Friedman HS, Prados MD, Wen PY, Mikkelsen T, Schiff D, Abrey LE, et al: Bevacizumab alone and in combination with irinotecan in recurrent glioblastoma. J Clin Oncol 27:47334740, 2009

9. Gilbert MR, Wang M, Aldape K, Stupp R, Hegi M, Jaeckle $\mathrm{KA}$, et al: RTOG 0525: A randomized phase III trial comparing standard adjuvant temozolomide (tmz) with a dose-dense (dd) schedule in newly diagnosed glioblastoma (GBM). Presented at the 2011 American Society of Clinical Oncology Annual Meeting, Chicago, 2011 (Abstract) (http://abstract. asco.org/AbstView_102_79659.html) [Accessed December $13,2012]$

10. Kerbel RS, Kamen BA: The anti-angiogenic basis of metronomic chemotherapy. Nat Rev Cancer 4:423-436, 2004

11. La Rocca RV, Mehdorn HM: Localized BCNU chemotherapy and the multimodal management of malignant glioma. Curr Med Res Opin 25:149-160, 2009

12. Macdonald DR, Cascino TL, Schold SC Jr, Cairncross JG: Response criteria for phase II studies of supratentorial malignant glioma. J Clin Oncol 8:1277-1280, 1990

13. Marras LC, Geerts WH, Perry JR: The risk of venous thromboembolism is increased throughout the course of malignant glioma: an evidence-based review. Cancer 89:640-646, 2000

14. McGirt MJ, Than KD, Weingart JD, Chaichana KL, Attenello FJ, Olivi A, et al: Gliadel (BCNU) wafer plus concomitant temozolomide therapy after primary resection of glioblastoma multiforme. Clinical article. J Neurosurg 110:583-588, 2009

15. Menei P, Metellus P, Parot-Schinkel E, Loiseau H, Capelle L, Jacquet G, et al: Biodegradable carmustine wafers (Gliadel) alone or in combination with chemoradiotherapy: the French experience. Ann Surg Oncol 17:1740-1746, 2010

16. Neyns B, Tosoni A, Hwu WJ, Reardon DA: Dose-dense temozolomide regimens: antitumor activity, toxicity, and immunomodulatory effects. Cancer 116:2868-2877, 2010

17. Noël G, Schott R, Froelich S, Gaub MP, Boyer P, Fischer-Lokou D, et al: Retrospective comparison of chemoradiotherapy followed by adjuvant chemotherapy, with or without prior Gliadel implantation (carmustine) after intial surgery in patients with newly diagnosed high-grade gliomas. Int J Radiat Oncol Biol Phys 82:749-755, 2012

18. Norden AD, Young GS, Setayesh K, Muzikansky A, Klufas $\mathrm{R}$, Ross GL, et al: Bevacizumab for recurrent malignant gliomas: efficacy, toxicity, and patterns of recurrence. Neurology 70:779-787, 2008

19. Pan E, Mitchell SB, Tsai JS: A retrospective study of the safety of BCNU wafers with concurrent temozolomide and radiotherapy and adjuvant temozolomide for newly diagnosed glioblastoma patients. J Neurooncol 88:353-357, 2008 


\section{Carmustine, temozolomide, and radiation therapy in glioblastoma}

20. Ryken T Carlisle T, Buatti J: Treatment of adults with newly diagnosed glioblastoma multiforme or anaplastic astrocytoma with surgery, gliadel wafers and limited field radiation plus concomitant temozolomide. J Clin Oncol 26 (Suppl):13003, 2008

21. Salvati M, D'elia A, Frati A, Brogna C, Santoro A, Delfini R: Safety and feasibility of the adjunct of local chemotherapy with biodegradable carmustine (BCNU) wafers to the standard multimodal approach to high grade gliomas at first diagnosis. J Neurosurg Sci 55:1-6, 2011

22. Sampson JH, Aldape KD, Archer GE, Coan A, Desjardins A, Friedman AH, et al: Greater chemotherapy-induced lymphopenia enhances tumor-specific immune responses that eliminate EGFRvIII-expressing tumor cells in patients with glioblastoma. Neuro Oncol 13:324-333, 2011

23. Simon R: Optimal two-stage designs for phase II clinical trials. Control Clin Trials 10:1-10, 1989

24. Stewart LA: Chemotherapy in adult high-grade glioma: a systematic review and meta-analysis of individual patient data from 12 randomised trials. Lancet 359:1011-1018, 2002

25. Stupp R, Mason WP, van den Bent MJ, Weller M, Fisher B, Taphoorn MJ, et al: Radiotherapy plus concomitant and adjuvant temozolomide for glioblastoma. N Engl J Med 352:987-996, 2005

26. Suzuki H, Itoh F, Toyota M, Kikuchi T, Kakiuchi H, Hinoda Y, et al: Quantitative DNA methylation analysis by fluorescent polymerase chain reaction single-strand conformation poly- morphism using an automated DNA sequencer. Electrophoresis 21:904-908, 2000

27. Tabatabai G, Stupp R, van den Bent MJ, Hegi ME, Tonn JC, Wick W, et al: Molecular diagnostics of gliomas: the clinical perspective. Acta Neuropathol 120:585-592, 2010

28. Tolcher AW, Gerson SL, Denis L, Geyer C, Hammond LA, Patnaik A, et al: Marked inactivation of O6-alkylguanineDNA alkyltransferase activity with protracted temozolomide schedules. Br J Cancer 88:1004-1011, 2003

29. Vredenburgh JJ, Desjardins A, Herndon JE II, Dowell JM, Reardon DA, Quinn JA, et al: Phase II trial of bevacizumab and irinotecan in recurrent malignant glioma. Clin Cancer Res 13:1253-1259, 2007

30. Westphal M, Hilt DC, Bortey E, Delavault P, Olivares R, Warnke PC, et al: A phase 3 trial of local chemotherapy with biodegradable carmustine (BCNU) wafers (Gliadel wafers) in patients with primary malignant glioma. Neuro Oncol 5: 79-88, 2003

Manuscript submitted January 16, 2012.

Accepted December 11, 2012.

Please include this information when citing this paper: published online January 25, 2013; DOI: 10.3171/2012.12.JNS111893.

Address correspondence to: Andrea Salmaggi, M.D., Fondazione Istituto Neurologico C. Besta, Via Celoria 11, Milan 20133 Italy. email: salmaggi@istituto-besta.it. 